\title{
Reflexiones Sobre Educación Inclusiva en Colombia: Estado De La Cuestión
}

${ }^{1}$.Institución Educativa Enrique Suárez, Colombia katherine.reina@uptc.edu.co 2.Universidad Pedagógica y

Tecnológica de Colombia

Como citar: Reina Avila, K., \& Lara Buitrago, P. (2020). Reflexiones en torno a la educación inclusiva en Colombia: estado de la cuestión. Educación y Ciencia, (24), e11381. https:// doi.org/10.19053/0120-7105. eyc.2020.24.e11381

\section{Resumen}

El presente artículo es una revisión documental de artículos académicos publicados en revistas especializadas, relacionados a la educación inclusiva y la enseñanza del inglés en Colombia en un período de tiempo comprendido entre 2006 y 2019. Se realizó un riguroso análisis de contenido con instrumentos de recolección de información como: fichas temáticas y matrices categoriales. Los resultados fueron agrupados en las siguientes categorías: concepto de educación inclusiva, legislación y normatividad, experiencias en contextos educativos, estudios sobre educación inclusiva y formas de enseñanza. Se concluye que, a pesar de los esfuerzos realizados por el gobierno colombiano, las instituciones educativas, los docentes e investigadores, aún se requiere mayor investigación sobre la enseñanza del inglés a estudiantes de inclusión para garantizar educación de calidad a esta población estudiantil.

Palabras clave: educación inclusiva, aprendizaje, enseñanza, educación bilingüe. 


\title{
Reflections about Inclusive Education in Colombia: State of topic
}

\begin{abstract}
This article is a documentary review of academic articles published in specialized journals, related to inclusive education and the teaching of English in Colombia, in a period of time between 2006 and 2019. A rigorous content analysis was carried out with instruments of information gathering such as: thematic files and categorical matrices. The results were grouped into the following categories: concept of inclusive education; legislation and regulations; experiences in educational contexts; studies on inclusive education and ways of teaching. It is concluded that, despite the efforts made by the Colombian government, educational institutions, teachers and researchers, more research is still required on the teaching of English to inclusion students to guarantee quality education to this student population.
\end{abstract}

Keywords: Inclusive education, learning, teaching, bilingual education.

\section{Introducción}

Históricamente las personas con discapacidad han sido calificadas como de menor valor al compararse con personas sin discapacidad, en este sentido, hacia finales del siglo XIX ya se encuentran evidencias de esta tendencia de juicio diferencial sobre el sujeto (Parra, 2011). Así, para atender a las particularidades de las personas con discapacidad, se adopta la caracterización de poblaciones, la cual plantea modelos educativos especiales para la sensibilización, responsabilidad e intervención de sujetos en condición de discapacidad. En parte, esta singularización poblacional permitió el surgimiento de instituciones, especialistas y el desarrollo de estudios sobre educación inclusiva. Siguiendo ese patrón de caracterización, a inicios del siglo XX, se socializa la primera prueba psicométrica que clasifica a los estudiantes de acuerdo a su nivel intelectual y en la cual, los resultados mostraron diferencias entre personas con discapacidad y aquellas que no. En consecuencia, toma fuerza la idea de una educación marginal para la población con discapacidad, sin embargo, el movimiento de normalización defendió la participación de todos los estudiantes en el sistema educativo y se opuso a un modelo de educación separatista. Estos antecedentes y el informe Warnock de 1978 marcaron el tránsito del término integración a inclusión

Finalizando el siglo XX, la educación inclusiva y la formación discursiva generan cambios en la escuela y el quehacer docente, así, la pedagogía activa y saberes como la biología, la psicología y la medicina, consolidan la enseñanza de los "anormales" (personas con discapacidad) (Orjuela, 2010, p. 224), posibilitando cambios estructurales en los procesos educativos. Autores como 
Cobos y Moreno (2014), Pérez (2016), y, Hernández y Velázquez (2016), señalan la educación inclusiva como un proceso heterogéneo, discontinuo y paradójico, gubernamentalizado bajo un gerencialismo que terceriza el servicio especializado (Yarza de los Ríos, 2011). Por su parte, Fernández y Duarte (2016) delimitan el encuentro y desencuentro de los términos asociados a la inclusión, en este sentido, definen de la integración, como la adaptación del estudiante al sistema educativo, $\mathrm{y}$, la inclusión como la adaptación del sistema educativo al estudiante. Es necesario destacar, que en Latinoamérica existe una amplia brecha y estigmas excluyentes sobre raza, condición y discapacidad, entre otros, lo cual acentúa la necesidad de incentivar las condiciones de acceso y calidad de la inclusión educativa para las personas con discapacidad, desde los diferentes entes gubernamentales (Acevedo, 2016; Blanco, 2004).

Este artículo, es resultado del estado de la cuestión de una investigación en curso que tiene como propósito definir la enseñanza del inglés a estudiantes de inclusión desde el concepto de hospitalidad, pretende dar cuenta de investigaciones referentes a la educación inclusiva y enseñanza del inglés en Colombia. El texto se estructura en los siguientes apartados: introducción, presentando el contenido del artículo; metodología, donde se detalla el proceso que permitió la elaboración del artículo. Posteriormente, los resultados muestran un recorrido por concepto de educación inclusiva, legislación y normatividad, experiencias en contextos educativos, estudios sobre educación inclusiva y estrategias pedagógicas para la enseñanza de la lengua extranjera. Finalmente se presentan las conclusiones sobre el estado del tema en cuestión.

\section{Metodología}

La investigación se desarrolla bajo el paradigma cualitativo usando el enfoque de investigación documental. La recolección y análisis de la información se logró empleando fichas temáticas y matrices de análisis que posteriormente se contrastaron a través de la técnica de revisión y análisis de contenido. Procedimentalmente, se rastrearon y seleccionaron 50 artículos académicos sobre inclusión en Colombia publicados entre los años 2006 a 2019, vinculando un número importante de publicaciones en 41 revistas especializadas. Para la búsqueda se usaron plataformas académicas especializadas como Scielo, Mendeley, Dialnet, Redalyc, Riberdis, Biblioteca virtual Universidad Pedagógica y Tecnológica de Colombia (UPTC), Biblioteca de la Universidad Externado y Hemeroteca de la Universidad Nacional Abierta y a Distancia (UNAD).

La selección de referencias y análisis documental siguió parámetros de clasificación y categorización que permitieron identificar y establecer tendencias de publicación en las siguientes áreas: concepto de educación inclusiva, legislación y normatividad, experiencias en contextos educativos, estudios sobre educación inclusiva y, formas de enseñanza . Estas categorías permitieron evidenciar y 
detallar los avances en la investigación sobre enseñanza del inglés a estudiantes de inclusión en Colombia.

\section{Resultados}

\section{Concepto Sobre Educación Inclusiva y Su Incidencia En Las Prácticas}

Las investigaciones sobre educación inclusiva muestran la evolución histórica del concepto, la modificación del término y producción de posibilidades para su comprensión en las dinámicas educativas. Agudelo M y Hurtado (2014) y Materón (2016) refieren que el concepto de inclusión se transformó desde exclusión, educación especial, educación integrada, hasta educación inclusiva, pasando por los términos anormalidad, necesidad especial, discapacidad y, diferencia, estos cambios conceptuales se pensaron en aras de promover la sana convivencia (Orjuela, 2010). Por su parte, Cardona (2013) plantea la educación inclusiva desde la tipología por cambios estructurales ligando la inclusión y la exclusión, en este sentido, argumenta la no clasificación de la diferencia teniendo en cuenta que no existen humanos iguales.

A partir de modificaciones de la Organización Mundial de la Salud, la Clasificación Internacional del Funcionamiento de las Discapacidades, la Organización de Estados Americanos, la Organización de Naciones Unidas, la Organización Panamericana de la Salud, la Declaración Universal de Derechos Humanos, el Pacto Internacional de Derechos Civiles y Políticos, la Convención de los Derechos del Niño y la Convención Interamericana para la Eliminación de todas las Formas de Discriminación de las Personas con Discapacidad y de los Estados de Salud, Pérez (2016), Materón (2016), Coy et al. (2013) y Padilla (2011), determinan que la educación inclusiva reúne limitaciones, deficiencias y restricciones que impiden la participación activa y dinámica en sociedad de las personas con discapacidad, solicitando que los sistemas educativos incluyan mejoras en su proceso.

De acuerdo con la clasificación internacional de funcionamiento, discapacidad y salud, la educación inclusiva se vincula con la capacidad de brindar un sistema educativo para todos que garantice ambientes de aprendizaje propicios en instituciones educativas (Vázquez, 2015). En consecuencia, Motta (2017) identifica tres tipos de necesidades respecto a las personas con discapacidad: educativas, educativas individuales y educativas especiales. Desde otra perspectiva, Robayo y Cárdenas (2017), encuentran en la inclusión un proceso y derecho que reconoce y suprime las barreras con apoyo de los docentes. Por su parte, Beltrán et al. (2015a) consideran que la educación inclusiva es un proceso orientado a la diversidad de características y necesidades del alumnado, incluyendo la escuela y los docentes. De acuerdo con Arnaiz (citado por Robayo \& Cárdenas, 2017), la educación inclusiva 
es la capacidad de una institución educativa de brindar equidad e igualdad de oportunidades de participación, las cuales deben estar enmarcadas en la legislación (Jaraba, 2015).

Respecto a las acciones gubernamentales sobre educación inclusiva, Vaillant (2011) enfatiza en que los procesos de capacitación y adecuación de políticas y lineamientos en América Latina, reflejan dos problemas, por un lado, el bajo estatus y capacitación inadecuada de los docentes, y por otro, la falta de cambios estructurales en políticas y lineamientos educativos. En esta línea, Calvo (2013) detalla la necesidad de generar oportunidades de igualdad y equidad en los procesos educativos, capacitación docente, así como, realizar cambios de políticas y currículo de las instituciones para de esta manera favorecer los procesos de educación inclusiva.

Así mismo, Canal (2014) sugiere modificar políticas públicas, currículo, estándares, sistemas de evaluación e indagar sobre bases científicas que respalden el derecho a la igualdad y educación para todos. Igualmente, se resalta que la educación inclusiva implica adaptar la educación a las condiciones individuales donde, además, se clasifica a los estudiantes en subgrupos para lograr la formación en paz (Coy et al., 2013). Finalmente, es necesario señalar que la terminología y los tratados a nivel internacional destacan acciones y prácticas para producir espacios inclusivos que permitan una educación encaminada al acceso y las condiciones apropiadas para responder a las necesidades de todos los estudiantes.

\section{Legislación y Normatividad Sobre Educación Inclusiva}

La normatividad sobre educación inclusiva fortalece la legitimidad, respaldo y apoyo de acciones relacionadas al acceso de la educación para todos. El siguiente apartado presenta una comparación de la normatividad sobre educación inclusiva en Colombia frente a otros países de Latinoamérica.

En Colombia, la educación superior implementa protocolos y normatividad frente a la educación inclusiva, supervisada por el Ministerio de Educación Nacional (MEN) (Beltrán et al., 2015b). Así mismo, en los niveles básica y media son las secretarías de educación y las entidades territoriales las encargadas de orientar los procesos respecto al tema, mientras que en Chile, es el Ministerio de Educación y la Unidad de Educación Especial, con ello, se aduce que en Colombia existe mayor número de intermediarios y actores trabajando los procesos relacionados a la educación inclusiva. Es preciso señalar, que Colombia y Chile han realizado cambios en políticas inclusivas basadas en el neoliberalismo (Vásquez, 2015). Por su parte, España orienta la atención a la diversidad teniendo en cuenta la Ley Orgánica 2/2006 y 4/2007, donde se estipula que son las comunidades autónomas las encargadas de la administración de los recursos. Sin embargo, ante este panorama 
es necesario destacar que se requieren mejoras de la calidad educativa, eficiencia, redistribución de la responsabilidad financiera y democratización en términos de educación inclusiva (Robayo \& Cárdenas, 2017).

Jaraba (2015) realiza una revisión en países latinoamericanos donde destaca que Argentina, cuenta con políticas inclusivas que garantizan el ingreso y gratuidad con el Programa Nacional de Becas Universitarias, además, asegura la inclusión al mundo laboral. De otro lado, Brasil desarrolla programas de ayuda económica y políticas contra la discriminación con el llamado sistema vestibular, igualmente, desde 2007 el Programa de Reestructuración y Expansión de las Universidades Federales permite la inclusión a la educación superior. Finalmente, México establece como derecho fundamental la equidad e igualdad educativa y para ello asigna un presupuesto estipulado, así mismo, crea el Programa Nacional De Fortalecimiento De La Educación Especial. Para la educación media superior, crea la Reforma Integral para la Educación Media Superior y un sistema de becas de excelencia (Jaraba, 2015). En consecuencia, es posible señalar que en América Latina existen centros, redes de apoyo, programas especiales y un presupuesto exclusivo que favorecen programas de educación inclusiva. No obstante, la población de inclusión fuera del sistema aún es significativa

En el mundo, el 15\% de la población sufre algún tipo de discapacidad, de esa cifra, 85 millones de personas están en América Latina (Rojas et al., 2018). De acuerdo al Departamento Administrativo Nacional de Estadísticas en 2006, Colombia presenta un $6.4 \%$ de población con discapacidad, lo que equivale alrededor de tres millones de ciudadanos. En consecuencia, existen organizaciones como la Institución Nacional para Ciegos, el Instituto Nacional para Sordos y la Federación Nacional de Sordos de Colombia, y el Instituto para la Rehabilitación Roosevelt, los cuales desarrollan y promueven procesos educativos de atención a la diversidad (Tilano-Vega et al., 2014). Igualmente, en 1981, con el Decreto 2358 nace el Sistema Nacional de Rehabilitación, y en 1985, el Instituto de Salud con la Resolución 14861 implementa normas para la protección de las personas con discapacidad, normativa que fue reafirmada en la Constitución Política de 1991 (Martínez et al., 2015).

Autores como Pérez (2016), Canal (2014), Hernández y Velázquez (2016) y Agudelo M y Hurtado (2016), resumen la normatividad colombiana teniendo como referente la Constitución Política de 1991, las leyes 115 de 1994, 361 de 1997, 1098 de 20061145 de 2007, 1346 del 2008, 1346 de 2009, Auto 006 de 2009, el documento CONPES 80, el Plan Nacional de Desarrollo y el Plan Nacional de Intervención en Discapacidad, definen que el estado debe garantizar la educación inclusiva de calidad a la población colombiana. En este sentido, los artículos 67 y 68 de la Constitución Política de 1991 legitiman el derecho de todos los colombianos a la educación, sin distinto alguno (Díaz \& Rodríguez, 2016). 
En 2009, la Vicepresidencia de la República de Colombia, el MEN, la subdirección de poblaciones y Bermúdez et al., conforman mesas de trabajo con el fin de generar lineamientos para la educación superior inclusiva. Del mismo modo, con el objetivo de establecer normativas que posibiliten una educación inclusiva, surge la Ley Estatutaria 1618 de 2013 y el Decreto 1421 de 2017 del MEN (Arias et al., 2016) y el Decreto 324 de 1996 el cual se adopta el lenguaje de señas y material audiovisual (Tilano-Vega et al., 2014). De otra parte, Correa y Rúa. (2018), Gómez (2015) y Cobos y Moreno (2014), señalan que se presentan tres tipos de educación: "la educación segregada, la educación integrada, y la educación inclusiva.” (Bermúdez et al., 2009, p. 99)

Según Arizabaleta y Ochoa (2016), Mosquera, Cárdenas y Nieto (2018), Robayo y Cárdenas (2017) y Acosta et al. (2017), el MEN evidencia que la tasa de deserción de personas vinculadas a programas de educación inclusiva $(45,3 \%)$ supera a la tasa de ingreso (42,3\%) y graduación de la educación superior (33,6\%). De otro lado, en 2013 el MEN promueve la gestión de la educación superior inclusiva mediante el Acuerdo 2034 por lo superior, así, se normativiza la educación inclusiva después de 1990 con parámetros de calidad, se promueve la no discriminación y la igualdad de condiciones educativas y oportunidades indican Acosta et al. (2017).

Por lo anterior, se aduce que los esfuerzos para garantizar la educación inclusiva en Colombia, radican en el acceso, gestión y seguimiento de los programas, lo cual, fortalece el reconocimiento nacional e internacional para la consecución de actividades que beneficien a la población con discapacidad. Estas acciones, se realizan teniendo en cuenta las características individuales de las personas para integrarse al sistema educativo, lo que es posible gracias a la aplicación de la normatividad estipulada por los gobiernos locales.

\section{Investigaciones Sobre Educación Inclusiva}

Sobre la metodología de investigación en educación inclusiva, Motta (2017) encuentra que corresponde a estudios cualitativos, cuantitativos, mixtos, etnográficos e históricos. En otra dirección, Fernández y Duarte (2016) realizan un estudio para determinar las facilidades académicas que brinda la UPTC a estudiantes de inclusión, en el cual se encuentra la falta de capacitación de los docentes y la necesidad de establecer una política inclusiva que fortalezca los programas educativos y las condiciones de inclusión. Por otra parte, Rojas et al. (2018) realizan un estudio en el que caracterizan estudiantes con discapacidades visuales, auditivas, motoras y de habla en la Facultad de Estudios a Distancia de la UPTC con el fin de evidenciar las oportunidades de las que disponen.

La oficina en Colombia del Alto Comisionado de las Naciones Unidas para los Derechos Humanos en compañía de investigadores de la Universidad Sergio Arboleda y Parra (2011) determinan que el proyecto denominado: Impacto de 
la Convención sobre los Derechos de las Personas con Discapacidad, desarrolla estrategias que permiten la educación de calidad para esta población. También, se resalta que el gobierno colombiano ha propendido por la mejora de condiciones para la educación inclusiva al generar leyes, asignar recursos y brindar apoyo de especialistas para favorecer el proceso educativo de estudiantes de inclusión.

\section{Experiencias de Educación Inclusiva en Colombia}

A continuación, se presentan diferentes estudios que se consideran experiencias en relación al trabajo con la inclusión en diversos escenarios educativos de básica primaria, secundaria, y educación superior.

Robayo y Cárdenas (2017) y Mosquera et al. (2018) indican que Colombia tiene un bajo presupuesto para la educación inclusiva, en estos términos, Acevedo (2016) presenta un estudio realizado en tres instituciones educativas de Bogotá donde se evidencia que los recursos físicos y humanos para atender a la población con discapacidades son insuficientes, y que algunas experiencias a pesar de las dificultades han aportado significativamente el contexto. Igualmente, en Medellín, Quinceno et al. (2016) señalan las transformaciones pedagógicas de docentes, por medio de talleres en los que fomentan planeaciones colaborativas, para de esta manera, garantizar una educación inclusiva de calidad.

Para integrar estudiantes con y sin discapacidad cognitiva en la escuela, Arias et al. (2016) desarrollan el proyecto Mosqueteros, donde analizan experiencias significativas de 2182 docentes de 65 municipios de Colombia entre los años 2012 y 2016. El estudio desarrollado por Agudelo G., et al. (2014) con el apoyo del MEN y el grupo de investigación Senderos del Instituto Tecnológico de Antioquia, pretende que personas en situación de vulnerabilidad accedan y permanezcan en el sistema educativo. De otra parte, Montaño y Vera (2012) revelan un estudio de caso realizado en una institución educativa de Bogotá y destacan la correcta redacción y aplicación de instrucciones, evaluación y actividades adaptadas a las necesidades de cada estudiante.

Por su parte, Alarcón et al. (2018) adelantaron un estudio vinculando 348 docentes de instituciones públicas de la ciudad de Cúcuta, donde identificaron la prevalencia de métodos de enseñanza adaptados al Proyecto Educativo Institucional. Así mismo, el proyecto de Beltrán et al. (2015b) hace referencia al mejoramiento de las condiciones educativas con una estrategia que los autores denominan aprendizaje dialógico, desarrollada en una institución educativa de carácter público.

De otra parte, Ramírez (2017) identifica que en la Institución Educativa Técnico Comercial de Jenesano (Boyacá) se han invertido recursos económicos suficientes que garantizan el acceso y calidad de la educación inclusiva para estudiantes, priorizando necesidades, metas y formas de pensar de estudiantes y padres de familia. También, en Caicedonia (Valle del Cauca), Acosta et al. (2017) encuentran 
que en el Colegio Bolivariano y Colegio Normal Superior los estudiantes no reciben la atención y recursos prometidos por el estado colombiano, lo cual, impide el desarrollo eficiente de procesos de educación inclusiva, sin embargo, es debido resaltar que docentes y directivos hacen su mayor esfuerzo para favorecer el proceso formativo de los estudiantes de inclusión.

Finalmente, González (2017) recoge testimonios de docentes colombianos acerca de la diversidad como manifestación y respeto por las diferencias, la homogeneización como barrera para la diversidad, y la situación de la inclusión y exclusión educativa en Colombia. Así, hace una crítica al gobierno por desatender la inconformidad de los docentes frente a las políticas del estado relacionadas al tema de educación inclusiva. Por ello, es necesario incrementar los esfuerzos, recursos y programas que permitan procesos inclusivos de calidad.

\section{Educación Superior Inclusiva}

Actualmente, la educación superior se enfoca a la gestión, transformación y adaptación de acciones inclusivas, además, potencia y valora la diversidad cultural, la formación de docentes y las investigaciones sobre inclusión (Arizabaleta \& Ochoa, 2016). Así, en 2007 el MEN lidera una revolución educativa superior con base en la ley 30 de 1992 generando criterios para cambiar estructuras de los lineamientos de educación superior (Yarza et al., 2013). Arizabaleta y Ochoa (2016) y Gómez (2015) afirman que en 2011 se identifica al grupo poblacional que requiere procesos de educación inclusiva, en 2012 se realiza el estudio conceptual y en 2013 se genera una política diferencial a partir de estrategias y acciones. La proyección hacia 2026 enfatiza en la educación para todos y la posibilidad de acceso, permanencia y graduación para el 2034 (Jaraba, 2015).

Igualmente, el Consejo Nacional de Acreditación promueve la gestión inclusiva en las Instituciones de Educación Superior determinando los factores, características e indicadores que permitan el acceso de los estudiantes, a través de políticas inclusivas de educación presencial y a distancia, no obstante, se requieren condiciones como conectividad, Tecnologías de la Información y Comunicación (TIC) y un tutor, para favorecer el acceso de la educación para todos. En el mismo sentido, Ballesteros et al. (2015) evidencian el uso de TIC en educación superior inclusiva y sus aportes en la enseñanza para facilitar el aprendizaje. No obstante, aquellos que no tienen acceso al recurso tecnológico representan un punto de quiebre en estos procesos (Jaraba, 2015). Finalmente, Martínez (2011) resalta la importancia de aplicaciones del e-learning, b-learning, m-learning y u-learning en el contexto de educación virtual, como herramientas que apoyan el proceso de aprendizaje.

Desde otra perspectiva, Acevedo (2016) destaca algunas experiencias de educación superior inclusiva a partir de la ética de la cultura organizacional 
multinivel y su importancia en relación a la diversidad, regida por manuales, marco legal e institucional, procedimientos y códigos de conducta. Por otra parte, aparece el programa de inclusión de la UNAD, el cual, tiene cinco características: participación, diversidad, interculturalidad, equidad y calidad. En algunas universidades se desarrollan proyectos que favorecen la educación inclusiva, como: TIRESIAS-Universidad de Antioquia, InclUtics-Universidad de San Buenaventura, así mismo, la Universidad del Cauca aporta a la interculturalidad con el proyecto de Alfabetización digital del resguardo de Guambia desarrollado en los años 2006 y 2007.

En la Universidad Autónoma de Bucaramanga, Pérez (2016) lleva a cabo un proyecto en el área metropolitana que aporta "prácticas innovadoras" y "procedimiento integral de atención e inclusión" (p. 127) y mejoran las condiciones educativas de los estudiantes de inclusión. En universidades bogotanas, Cobos y Moreno (2014) identifican programas, proyectos, dependencias y adaptación del currículo con base en condiciones especiales. Por ello, recomiendan implementar ajustes razonables, diseño universal de aprendizaje y crear dependencias enfocadas en atender la discapacidad y favorecer la inclusión.

Finalmente, Romero y Urrego (2016) encuentran que las universidades han diseñado planes de estudio adaptados que favorecen la educación inclusiva, además, reconocen al docente como el principal agente estimulador y promotor del aprendizaje de estudiantes con discapacidad auditiva. Igualmente, la Facultad de Estudios a Distancia de la UPTC, usa el software: Herramientas Tecnológicas para Ayuda Humanitaria que traduce mensajes a lenguaje de señas, lo cual favorece y garantiza el derecho a la educación de las personas con discapacidad auditiva (Rojas et al., 2018).

\section{Formas de Enseñanza y Educación Inclusiva}

En este punto, es importante destacar la importancia de indagar y conocer sobre las formas de enseñanza en educación inclusiva, pues permite considerar reflexiones para tener proyecciones y antecedentes desde donde establecer puntos de partida en el planteamiento de acciones inclusivas en los escenarios educativos, en tanto, para esta revisión se situaron las consideraciones del tema abordado en el área de la enseñanza de lengua extranjera.

De esta manera, Arizabaleta y Ochoa (2016) retoman a Porter para definir las estrategias de enseñanza de un segundo idioma, teniendo en cuenta los diagnósticos, análisis de las instituciones, descripción de necesidades de los programas educativos, implementación de lineamientos y estrategias pedagógicas. Por su parte, Gutiérrez (2017) propone la dialogicidad como una herramienta para el desarrollo de sociedades inclusivas, acercamiento intercultural y comprensión de la realidad del otro. Así mismo, se reconoce que los enfoques de aprendizaje colaborativo, 
autónomo, tutoría, recursos multimedia, aprendizaje basado en tareas y traducción gramatical, son aptos para la educación inclusiva, desde la perspectiva de Mosquera et al. (2018).

Por su parte, Alfaro (2015) se remite al programa Ondas, el cual propone una estrategia pedagógica basada en la investigación, para resaltar la capacidad de inclusión y aceptación de estudiantes de la etnia wayuu. De otro lado, la estrategia día a día, que hace referencia a: DÍA (Diagnosticar, Identificar, Adaptar) A (articulado a) DÍA (Dedicación ilimitada, Integración sociocultural, Atención personalizada), ejecuta acciones inclusivas. A manera particular, Rivera de Antonio (2017) fomenta el trabajo colaborativo entre docentes, estudiantes y la familia de un estudiante con Asperger. Así mismo, Pérez (2016) y Alfaro (2015), fomentan el civismo y las prácticas inclusivas en los estudiantes, docentes, directivos y padres de familia, con actividades académicas y formativas estandarizadas.

Otra estrategia frecuente para garantizar calidad en los procesos de educación inclusiva es la planeación pedagógica adaptada a las necesidades y contexto de cada estudiante (Quinceno et al., 2016). Según Acevedo (2014), es necesario el sujeto en contexto, el registro de historias de vida, la identificación de necesidades, la búsqueda de antecedentes, el establecimiento de condiciones tecnológicas y hallazgo de la mejor solución, por ejemplo, la Norma Técnica 5854 establece principios para la implementación de páginas web.

En 1994, la Ley General de Educación estipula la importancia del inglés en la globalización y en 2004 establece el Programa Nacional de Bilingüismo (PNB). En esta dirección, Robayo y Cárdenas (2017) encuentran que la implementación del PNB en Colombia y sus estándares de aplicación, se enfocan en la globalización, sin respetar las necesidades de los estudiantes de inclusión. Igualmente, no se encuentra una guía que permita la enseñanza del inglés a estudiantes de inclusión (Mosquera et al., 2018). Es preciso destacar, que el MEN en 2007 estableció estándares básicos de competencias para generar estrategias de enseñanza y flexibilización del Plan Educativo Institucional y currículos.

\section{Conclusiones}

Con el paso de los años, el concepto de educación inclusiva sufre cambios estructurales que permiten mejorar la prestación del servicio educativo. En sus comienzos, se dio un contexto clínico a la educación inclusiva, lo cual, generó discriminación y rechazo. Luego, el sistema educativo busca herramientas y estrategias que integran y direccionan el proceso educativo a la adaptación del estudiante a la escuela, de esta manera, se logró llegar a la integración y aceptación de las diferencias en la escuela, lo que implica adaptarse a las necesidades de cada estudiante, con ello, desarrollar procesos de educación inclusiva.

En Latinoamérica, los gobiernos de Argentina, Chile, México, Brasil y Colombia 
realizan modificaciones de sus leyes, lo que garantiza brindar condiciones de igualdad de oportunidades desde la básica primaria hasta la educación superior. Así mismo, desarrollan estrategias y normativas que crean instituciones especializadas, capacitan a personal de apoyo, contrata personal especializado, aumenta el presupuesto, y, apoya con material y adecuaciones locativas a las instituciones educativas. Sin embargo, es necesario evitar la tercerización en la contratación de personal especializado, pues, al ser un agente ajeno a la institución, no puede dar cuenta de la realidad del proceso educativo de los estudiantes de inclusión.

En Colombia, se han desarrollado investigaciones que reflejan que, a pesar de los esfuerzos del gobierno por mejorar las condiciones educativas de los estudiantes de inclusión, se deben aumentar los recursos físicos, locativos y humanos para garantizar calidad en el proceso y los resultados. Así mismo, se destaca la acción de los docentes de instituciones educativas públicas que generan sus propias estrategias de enseñanza adecuadas a las necesidades y contexto de los estudiantes, no obstante, se requiere mayor investigación en el campo de la enseñanza de lengua extranjera a estudiantes de inclusión para brindar mejores condiciones educativas.

Con relación a la educación superior, se concluye que las universidades requieren mejoras significativas en el manejo de la educación inclusiva, para brindar condiciones que favorezcan el proceso educativo. De igual modo, Fernández y Duarte (2016) evidencian que el sistema funciona en el papel pero no en la realidad, debido a que los docentes no reciben capacitación suficiente y la adecuación de los currículos se realiza de manera improvisada a medida que surge la necesidad.

Finalmente, es preciso señalar que para que el proceso de educación inclusiva logre los resultados esperados por el gobierno colombiano, se necesita coherencia entre lo escrito y las acciones, de esta manera, se orientará hacia una misma dirección que permita mejorar las condiciones educativas de los estudiantes, y así, el proceso de enseñanza y aprendizaje sea significativo y garantice su integración en la sociedad.

\section{Referencias}

Acevedo, S. (2014). Inclusión digital y educación inclusiva. Aportes para el diseño de proyectos pedagógicos con el uso de tecnologías de la comunicación. Revista de investigaciones UNAD, 13(1), 41-57. http://hemeroteca.unad.edu.co/index. $\mathrm{php/revista-de-investigaciones-unad/article/view/1130/1328}$

Acevedo, S. (2016). Reflexiones éticas sobre gestión de la diversidad en educación superior inclusiva. Praxis \& saber, 7(15), 147 - 164. http://dx.doi. org/10.19053/22160159.v7.n15.2016.5727

Acosta, L., Lugo, J., \& Solano, F. (2017). Educación inclusiva en las instituciones educativas de Caicedonia Valle del Cauca, Colombia. Prospectiva, (25), 113-140. 
http://www.scielo.org.co/pdf/prsp/n25/2389-993X-prsp-25-00113.pdf

Agudelo, G., Bedoya, M., \& Correa, J. (2014). Formación de docentes participantes en el programa de educación inclusiva con calidad en Colombia. Revista latinoamericana de educación inclusiva, (9), 43-61. https://www.mendeley.com/ catalogue/f4555324-b8a3-3675-8844-4f311ddaf6b5/

Agudelo, M., \& Hurtado, L. (2014). Inclusión educativa de las personas con discapacidad en Colombia. Movimiento y salud, 2(1), 45-55. http://riberdis.cedd. net/handle/11181/4889

Alarcón, M., Bonilla, N., Carrillo, S., Forgiony, J., Montanchez, M., \& Rivera Porras, D. (2018). Prácticas pedagógicas frente a la educación inclusiva desde una perspectiva docente. Espacios, 39(17), 15-33.

Alfaro, C. (2015). La investigación como estrategia pedagógica en la Guajira, desde una perspectiva de la inclusividad y diversidad como nuevo paradigma para el desarrollo de habilidades, destrezas y competencias. Educación y ciudad, (29), 150162. http://repositoryinst.uniguajira.edu.co:8080/xmlui/handle/123456789/53

Arias, L., Montoya, M., \& Zuluaga, A. (2016). Proyecto mosqueteros: una propuesta de intervención en el aula para favorecer los procesos de inclusión escolar. Pensamiento psicológico, 14(1), 77-88. http://dx.doi.org/10.11144/Javerianacali. PPSI14-1.pmpi

Arizabaleta, M., \& Ochoa, A. (2016). Hacia una educación superior inclusiva en Colombia. Pedagogia y saberes (45), 41 - 52.

Ballesteros, B., Iriarte, F., Jabba, D., Ordoñez, M., Ricardo, C., Said, E., \& Vergara, E. (2015). Fortalecimiento pedagógico en las universidades en Colombia a través de las TIC. Caso de la región Caribe. Educación XX1, 18(2), 277-304.

Beltrán, Y., Martínez, Y., \& Vargas, B. (2015a). El sistema educativo colombiano en camino hacia la inclusión: avances y retos. Educación y educadores, (18), 65-72. http://dx.doi.org/10.5294/edu.2015.18.1.4

Beltrán, Y., Martínez, Y., \& Torrado, O. (2015b). Creación de una comunidad de aprendizaje: una experiencia de educación inclusiva en Colombia. Encuentros, 13(2), 57-72. http://dx.doi.org/10.5569/2340-5104.02.02.05

Bermúdez, G., Cuervo, G., Flórez, R., \& Moreno, M. (2009). Lineamientos de política para la atención educativa de poblaciones en situación de discapacidad en las instituciones de educación superior en Colombia. Fonoandiología Iberoamericana, (9), 11-24. https://arete.ibero.edu.co/article/view/393

Blanco, R. (2004). La equidad y la inclusión social: uno de los desafíos de la educación y la escuela de hoy. Revista Electrónica Iberoamericana sobre calidad, eficacia y cambio en educación, (4), 1696-4713. 
Calvo, G. (2013). La formación de docentes para la inclusión educativa. Páginas de educación, 6(1), 13-33.

Canal, J. (2014). Discapacidades en la escuela. Revista de Investigación Silogismo, 1(14), 116-120.

Cardona, H. (2013). Perspectiva topológica de la inclusión en la educación superior. Espiral, Revista de Docencia e investigación, 3(2), 41-52.

Cobos, A., \& Moreno, M. (2014) Educación superior y discapacidad: análisis desde la experiencia de algunas universidades colombianas. Revista española de discapacidad, (2), 93-101.

Correa, L., \& Rúa, J. (2018). La trampa de la educación especial: rodeos y laberintos jurisprudenciales para garantizar el derecho a la educación inclusiva de las personas con discapacidad. Revista de derecho del estado, (41), 97-118.h ttps://doi. org/10.18601/01229893.n41.04

Coy, L., Martín, E., \& Sarmiento, P. (2013). Educación inclusiva y diversidad funcional en la universidad. Revista facultad de medicina, 61 (2), 195 - 204. http:// riberdis.cedd.net/handle/11181/4525

Díaz, L., \& Rodríguez, L. (2016). Educación inclusiva y diversidad funcional: conociendo realidades, transformando paradigmas y aportando elementos en la práctica. Revista del instituto de estudios educación universidad del norte, (24), 43-61. http://dx.doi.org/10.14482/zp.24.8721

Fernández, F., \& Duarte, J. (2016). Retos de la inclusión académica de personas con discapacidad en una universidad pública de Colombia. Formación universitaria,? (4), 95-104. http://dx.doi.org/10.4067/S0718-50062016000400011

Gómez, M. (2015). Derecho a la educación superior inclusiva para grupos de especial protección. Educación y desarrollo social, (9), 10-27.

González, M. (2017). Diálogos de saberes. Las homogeneizaciones-diversidades y las exclusiones-inclusiones en la educación colombiana, narrativas autobiográficas. Revista de pedagogía, (38), 211-248.

Gutiérrez, M. (2017). Repensar el papel del diálogo para la inclusión social, la responsabilidad política y la educación dialógica. Actualidades pedagógicas, (69), 15-47. http://dx.doi.org/10.19052/ap.3765

Hernández, E., \& Velásquez, J. (2016). Comprensiones del discurso normativo sobre inclusión educativa en Colombia. El Ágora U.S.B., 16(2), 493-512.

Jaraba, L. (2015). Experiencias relacionadas con la implementación de políticas de inclusión social en educación a distancia. Espiral, (5), 111-126. http://dx.doi. org/10.15332/erdi 
Martínez, A., Uribe, A., \& Velazquez, H. (2015). La discapacidad y su estado actual en la legislación colombiana. Duazary, (12), 49-58.

Materón, S. (2016). Principios de equidad e igualdad: una perspectiva inclusiva para la atención educativa de las poblaciones con discapacidad en Colombia. Revista colombiana de bioética. 11(1), 25-153. https://doi.org/10.18270/rcb.v11i1.1817

Montaño, J., \& Vera, E. (2012). Inclusive English classrooms: requirements, implications and limitations. -A qualitative case study-. Folios, (36), 77-95.

Mosquera, O., Cárdenas, M., \& Nieto, M. (2018). Pedagogical and research approaches in inclusive education in ELT in Colombia: Perspectives from some Profile journal authors. Profile: Issues in Teachers' Professional Development, (20), 231246. https://doi.org/10.15446/profile.v20n2.72992.

Motta, E. (2017) Caracterización de estudios e investigaciones sobre la relación entre necesidades educativas especiales con población sorda y representaciones sociales desde la inclusión. Actualidades pedagógicas, (70), 97-121. http://dx.doi. org/10.19052/ap.4109

Orjuela, G. (2010) El interés por la diferencia en el mundo contemporáneo: ¿Una posibilidad para pensar hoy la educación especial?. Praxis \& Saber. 1(1), 217-233.

Padilla, A. (2011). Inclusión educativa de personas con discapacidad. Revista colombiana de Psiquiatría, 40(4), 670-699.

Parra, C. (2011). Educación inclusiva en un modelo de diversidad humana. Educación y desarrollo social, 5(1), 139-150.

Pérez, L. (2016). Praxis inclusiva, para la atención de necesidades educativas especiales en el sector rural de Bucaramanga. Praxis \& saber, 7(15). 127 - 145. http://dx.doi.org/10.19053/22160159.v7.n15.2016.5726

Quinceno, E., Tamayo, W., \& Velásquez, Y. (2016). Construcción de planeaciones pedagógicas para la educación inclusiva. Actualidades investigativas en educación, 16(3), 1-35. http://dx.doi.org/10.15517/aie.v16i3.26113

Ramírez, W. (2017). La inclusión: una historia de exclusión en el proceso de enseñanza-aprendizaje. Cuadernos de lingüistica hispánica, (30), 211-230.

Rivera de Antonio, F. (2017). El síndrome asperger: estrategias, soluciones para una inclusión efectiva. Actualidades pedagógicas, (69), 133-165. http://dx.doi. org/10.19052/ap.4078

Robayo, L. \& Cárdenas, M. (2017). Inclusive education and ELT policies in Colombia: Views from some PROFILE journal authors. PROFILE Issues in Teachers' Professional Development, (19), 121-136. https://www.crossref.org/ iPage?doi=10.15446\%2Fprofile.v19n1.61075 
Romero, M., \& Urrego, S. (2016). Inclusión de personas con discapacidad sensorial (ciegos y sordos) en los programas de formación musical universitaria en la ciudad de Bogotá, D.C. Uni-pluri/versidad, 16(2), 27-40.

Rojas, L., Arboleda, N., \& Pinzón, L. (2018). Caracterización de población con discapacidad visual, auditiva, de habla y motora para su vinculación a programas de pregrado a distancia de una universidad de Colombia. Revista Electrónica Educare, 22(1), 1-28. http://dx.doi.org/10.15359/ree.22-1.6

Tilano-Vega, L., Cardenas-Torres, A., Betancur-Caro, I. Rivera-Muñoz, L., GómezGómez, B., Arango-Restrepo, J., Moreno-Asprilla, R., \& Jaramillo-Velázquez, A. (2014). Tools facilitating communication for the deaf. Educación y Educadores, (17), 468-480. http://dx.doi.org /10.5294/edu.2014.17.3.4

Vaillant, D. (2011). Preparing teachers for inclusive education in Latin America. Prospects, 137(1). 385. http://dx.doi.org/10.1007/s11125-011-9196-4

Vásquez, D. (2015). Políticas de educación inclusiva: Una comparación entre Colombia y Chile. Educación y educadores, (18), 45-61. http://dx.doi.org /10.5294/ edu.2015.18.1.3

Yarza, A., Rojas, H., \& López, J. (2013). Discapacidad y educación superior: las contiendas por la participación y la ciudadanía de las reformas neoliberales de la universidad y la educación superior en Colombia. Revista latinoamericana de educación inclusiva, 8(1), 35-49. http://ayura.udea.edu.co:8080/jspui/ handle/123456789/3175

Yarza de los Ríos, V. (2011). Educadores especiales en la educación inclusiva como reforma y práctica de gubernamentalidad en Colombia: ¿perfil personal o productor de saber? Curriculum sem fronteiras, 11(1), 34-41. http://200.24.17.10/ handle/10495/3924 